\title{
MICROPROBLEMATICA DEL CARBONÍFERO INFERIOR DEL ÁREA DEL GUADIATO (SUROESTE DE ESPAÑA)
}

\author{
Pedro CÓZAR y Sergio RODRÍGUEZ
}

\author{
Departamento y UEI de Paleontología, Facultad de Ciencias Geológicas, \\ Universidad Complutense de Madrid, Ciudad Universitaria s/n., 28040-Madrid, E- \\ mail: Sergrodr@eucmax.sim.ucm.es
}

Cózar, P. y Rodríguez, S. 2000. Microproblematica del Carbonífero inferior del Área del Guadiato (suroeste de España). [Lower Carboniferous Microproblematica from Guadiato Area (southwestern Spain).] Revista Española de Paleontología, 15(2), 105-116. ISSN 0213-6937.

\begin{abstract}
Part of the Lower Carboniferous microfossil assemblages from the Guadiato Area, between the Osa-Morena and Central Iberian Zones are discussed, viz. forms of doubtful affiliation. Some taxa can probably be refered to the foraminifera: Gibasbia Strank and Paracaligelloides Reitlinger. Some other have a more doubtful position between foraminifera, volcovaceans and algae: Diplosphaerina (Derville) emend. Conil et al., Polysphaerinella Mamet, Parathurammina Suleimanov, and Hemithurammina Mamet. A third group may correspond to dasycladean algal parts or volvocaceans: Calcisphaera Williamson emend. Andrews, Paleocancellus Derville and Asterosphaera Reitlinger. Finally, the available information on two genera, Draffania Cummings and Saccamminopsis Sollas, does not allow to affiliate them to any precise group.
\end{abstract}

Keywords: Microproblematica, Parathuramminacea, late Viséan, early Serpukhovian, SW Spain.

\section{RESUMEN}

Este trabajo está basado en parte de las asociaciones de microfósiles del Área del Guadiato, en el Carbonífero inferior, entre las zonas de Ossa-Morena y Centro-Ibérica. Se han agrupado aquellos taxones cuya asignación es dudosa. Algunos de ellos son probablemente asimilables a foraminíferos: Gigasbia Strank y Paracaligelloides Reitlinger. Otros tienen una posición más dudosa, entre foraminíferos, volvocales, o partes de algas: Diplosphaerina (Derville) emend. Conil et al., Polysphaerinella Mamet, Parathurammina Suleimanov y Hemithurammina Mamet. Un tercer grupo podría corresponder a partes de algas dasicladáceas o volvocales: Calcisphaera Williamson emend. Andrews, Paleocancellus Derville y Asterosphaera Reitlinger. Por último, la información disponible sobre dos géneros, Draffania Cummings y Saccamminopsis Sollas, no permite aproximarlos a ningún grupo concreto.

Palabras clave: Microproblematica, Parathuramminacea, Viseense superior, Serpukhoviense inferior, SO España.

\section{INTRODUCCIÓN}

En el Área del Guadiato afloran rocas del Carbonífero inferior, en el límite entre las zonas de Ossa-Morena y Centro-Ibérica (Fig. 1), en la provincia de Córdoba. Los afloramientos fueron divididos en tres unidades estructurales (Fig. 2): Unidad del Fresnedoso, U. Sierra del Castillo y U. San Antonio-La Juliana (Cózar y Rodríguez, 1999). En los materiales se ha reconocido el Viseense superior y Serpukhoviense inferior, siendo identificadas las zonas de foraminíferos 14 a 18 propuestas por Mamet (1974).

En este trabajo se describen una serie de formas tradicionalmente incluidas en los foraminíferos, en diversas familias, tales como Archaesphaeridae, Parathuramminidae, Saccamminidae, etc. (ver clasificaciones de Loeblich y Tappan, 1964; Loeblich y Tappan, 1988; Vachard, 1994). Sin embargo, la relación de estos taxones con los foraminíferos es dudosa por razones diversas, ya sea por la estructura de la pared, por la similitud con determinadas formas de afinidad conocida no pertenecientes a foraminíferos actuales, etc. Otra característica considerada y que no tiene nada que ver con la propia morfología de los taxones, está relacionada con los condicionantes paleoecológicos, ya que es común que las rocas originadas en lagoones restringidos contengan un elevado número de ejemplares de estos microproblemática (Vachard, 1994). Estos 


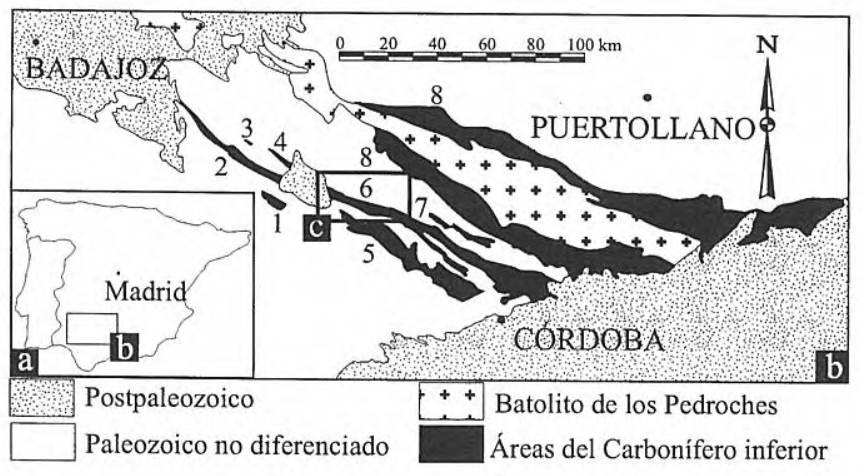

Figura 1. Localización de la zona de estudio. A Península Ibérica. B: Áreas que se consideran incluidas en la Cuenca del Carbonífero inferior de la zona Norte de Ossa-Morena, 1. Berlanga, 2. Matachel, 3. El Casar, 4. Campillo de Llerena, 5. Benajarafe, 6. Guadiato-Guadalmellato, 7. Guadalbarbo, 8. Pedroches. C: Sector Norte del Área del Guadiato (modificado de Cózar y Rodríguez, 1999).

Location of the studied area. A: Iberian Peninsula. B: Areas included in the northern zone of the Lower Carboniferous Ossa-Morena Basin, 1. Berlanga, 2. Matachel, 3. El Casar, 4. Campillo de Llerena, 5. Benajarafe, 6. GuadiatoGuadalmellato, 7. Guadalbarbo, 8. Pedroches. C: North sector of the Guadiato Area (modified from Cózar and Rodríguez, 1999).

niveles con tal abundancia de ejemplares no pueden ser el resultado de la acumulación de foraminíferos, ya que tal concentración implicaría un alto nivel de nutrientes que es difícil suponer en este tipo de medios. Por ello es más probable que se trate de algunas partes de algas, quistes u otros elementos de las mismas. Las algas, por su tipo de alimentación autótrofa, no dependen en la misma medida de los nutrientes externos.

Las variaciones en la posición taxonómica que han sufrido algunos de estos taxones son grandes. Un caso conocido en la bibliografía es el de las formas citadas como Radiosphaera, que posteriormente se comprobó que correspondían a "quistes" individualizados de un alga, Radiosphaeroporella Mamet y Boulvain, 1992. En las rocas del Guadiato se ha encontrado esta alga, tanto partes del talo como "quistes" individualizados.

El objetivo de este trabajo no es establecer una propuesta de posición taxonómica para los distintos taxones. Solamente se indica dónde han sido situados por autores anteriores, se exponen los datos observados en las asociaciones del Guadiato, y, en algún caso, la afinidad más probable que hemos supuesto a la vista de los datos observados. Finalmente, se realiza una breve descripción de los taxones.

En la Fig. 3 se expone la distribución de estos taxones en el Área del Guadiato. En vista de los resultados obtenidos en la región, ninguno de ellos tiene una gran utilidad bioestratigráfica, ya que el reconocimiento de las zonas de la Fig. 3 está basado en las asociaciones de foraminíferos, pertenecientes fundamentalmente a Endothyroida, que se pueden ver en Cózar Maldonado (1998).

Las asociaciones de Microproblematica han sido obtenidas a partir del estudio de unas 1800 láminas delgadas, realizadas en unas 50 secciones estratigráficas en el Área del Guadiato (Cózar Maldonado, 1998). Todas las láminas delgadas en las que está basado este estudio están depositadas en el Departamento de Paleontología de la Facultad de Ciencias Geológicas (Universidad Complutense de Madrid). Las siglas que aparecen en cada una de las figuras corresponden a las secciones estratigráficas, tramo de la sección y número de ejemplar. Las abreviaturas utilizadas son: ADF (Adelfilla), BUJ (Burjadillo), CAR (Caridad), NVC (Navacastillo), SPL1 (Sierra Palacios 1), SPL7 (Sierra Palacios 7), PÑR2 (Peñarroya 2), PÑR5 (Peñarroya 5), SSE1 (Sierra de la Estrella 1), y SSE2 (Sierra de la Estrella 2). La localización de estas secciones estratigráficas se encuentra en Cózar Maldonado (1998).

\section{CONSIIDERACIONES TAXONÓMICAS}

El mayor problema a la hora de estudiar estas formas microproblemáticas es la incertidumbre de su posición taxonómica, y, por tanto, interpretar cual era la función de cada una de las estructuras que se observan ("cámaras", espinas, poros, etc.).

Tradicionalmente se agrupaban en la superfamilia Parathuramminacea, pero el análisis de los distintos géneros debe hacerse de una forma individualizada, ya que dentro de esta superfamilia hay formas muy semejantes, pero que pueden tener un origen totalmente distinto.

Los géneros Calcisphaera, Paleocancellus y Asterosphaera se pueden agrupar bajo el calificativo de calcisferas, muy ampliamente extendido en la literatura. Estos taxones fueron originalmente interpretados como radiolarios carboníferos (Judd, 1877), pero a partir de Williamson (1881) se relacionaron con las algas. Muchos autores siguieron esta hipótesis (i.e. Cayeux, 1929; Derville, 1931, 1952), relacionando estas esferas con esporas de "algas azul-verdosas". Posteriormente Rupp (1966) mostró quistes calcificados de dasicladáceas actuales, semejantes a las calcisferas fósiles. Mamet (1973) figura un "anillo" de calcisferas alrededor de un "agujero" central. Mamet (1998) define una nueva alga dasicladácea, Ningbingellina, del Devónico de Australia, con grandes similitudes con el anillo constituido por Calcisphaera laevis Williamson, 1881, aunque ambas formas se diferencian en que el "anillo" de calcisferas no tiene calcificados los bordes que conectan las esferas, ni se observan suturas rectas entre ellas, como es el caso de Ningbingellina o de Radiosphaeroporella.

Por el contrario, Vachard y Tellez (1986) enumeran hasta nueve criterios por los que las calcisferas no pueden asimilarse a los quistes de dasicladáceas, y también descartan su inclusión en otros grupos, como dinoflagelados, foraminíferos, quistes de crisofitas, etc. 


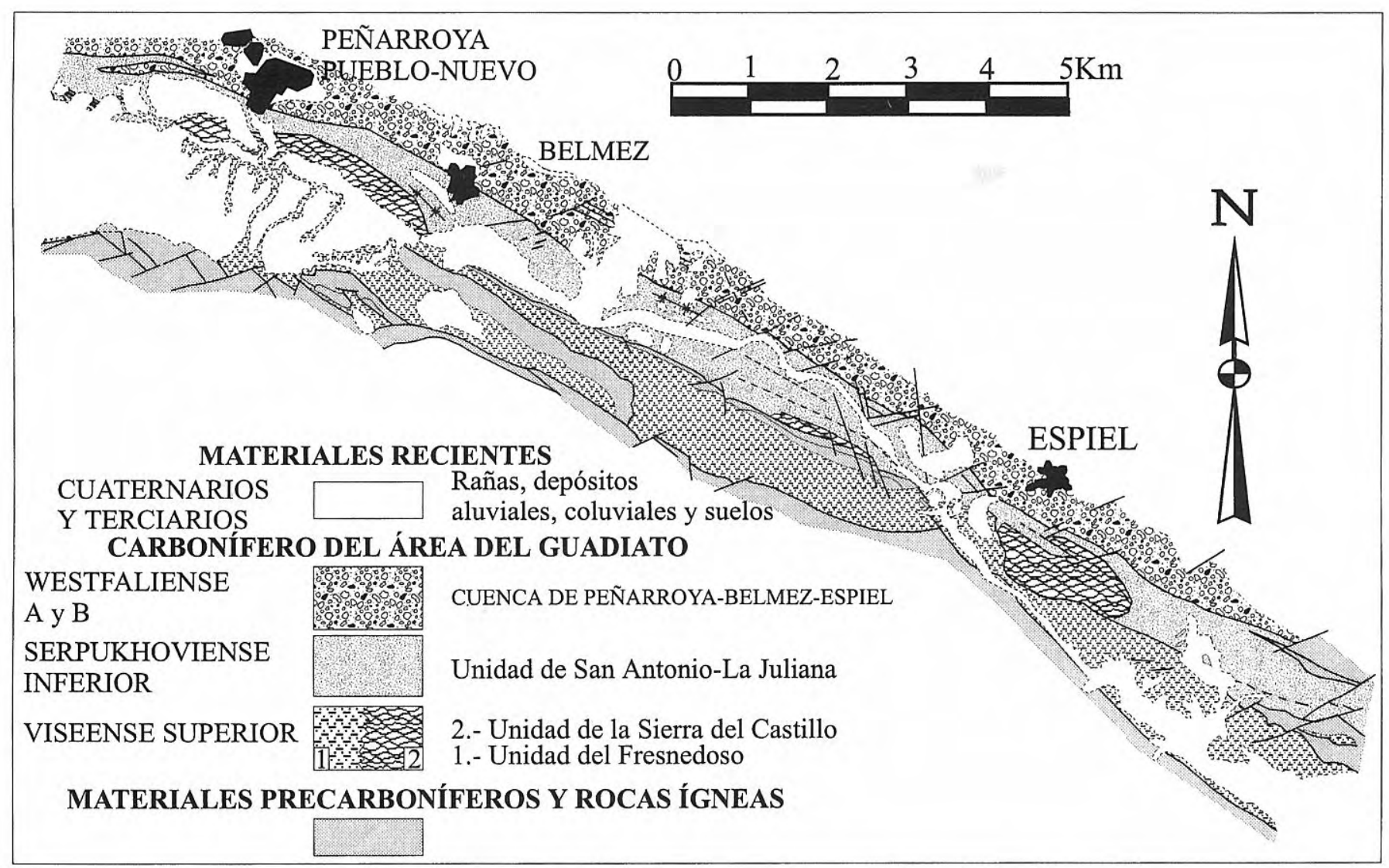

Figura 2. Sector Norte del Área del Guadiato, detalle de la Fig. 1C (modificado de Cózar y Rodríguez, 1999). North sector of the Guadiato Area, Fig. 1C in detail (modified from Cózar and Rodríguez, 1999).

Estos autores se basan en los trabajos de Kazmierczack (1975, 1976 y 1981) para inferir una relación de las calcisferas con las volvocales actuales y del Precámbrico. Otro problema sería la asignación de las volvocales actuales, en la que tampoco hay un acuerdo entre los diferentes autores (ver discusión en Vachard y Tellez, 1986).

Parathurammina y Hemithurammina han sido clásicamente atribuidos a los protozoos, incluidos dentro de la familia Parathuramminidae. Existen pocos trabajos en los que se dude de esta posición taxonómica, como es el de Armstrong y Mamet (1977). En este caso, al igual que en las calcisferas, su distribución paleoecológica es totalmente contradictoria con un origen relacionado con los protozoos, ya que aparecen concentrados en lagoones (Armstrong y Mamet, 1977; Vachard, 1994). Esto sugiere que están más relacionados con organismos de alimentación autótrofa, como puede ser con "quistes" calcificados de algas (Armstrong y Mamet, 1977). Aunque estos argumentos paleoecológicos parecen suficientes para negar la relación con los protozoos, existen otros datos que deben ser tenidos en cuenta:

- Mamet (com. pers.) ha observado estructuras rígidas que formaban "armazones" tridimensionales. Aunque éstos se podrían interpretar también como quistes sicigiales reproductivos, como lo han sido algunos "armazones" constituidos por foraminíferos en el Carbonífero (Groves, 1987, 1988; Villa y Sánchez de Posada, 1991). Pero los "armazones" de Parathurammina parecen totalmente diferentes y con una utilidad distinta (¿soporte de una estructura?).

- El disco basal que se observa en Hemithurammina puede estar curvado o plano, y, en muchas ocasiones, los ejemplares aparecen incrustados (Fig. 4K) sobre cualquier tipo de bioclasto (alga, briozoo, etc.). Parece claro que este tipo de incrustamiento no es posible en cualquier tipo de quiste algal, sino en entidades fisiológicamente completas (¿protozoos?).

Diplosphaerina es una forma sobre la que son habituales los estudios poblacionales. Conil et al. (1973) consideraron cuatro géneros como representantes de los diferentes estadios dentro de la vida de un mismo organismo: Archaesphaera Suleimanov, 1945, Diplosphaerina Derville, 1952, Eotuberitina MiklukhoMaclay, 1958, y Neotuberitina Miklukho-Maclay, 1958; siendo Archaesphaera el que tiene prioridad, y caracterizándola con cuatro estadios evolutivos: esférico (S), distendido (SD), diplosférico (D) y tuberitínido (T). Al reunificar estos géneros, los autores asumen que es posible que en las especies más antiguas en un estadio esférico (S) (Archaesphaera minima Suleimanov, 1945) la pared sea estrecha y aparentemente no perforada, mientras que el resto de especies sí tienen la pared perforada.

De forma análoga, Browne y Pohl (1973) llegan a la misma conclusión, pero estos autores incluyen también Tuberitina Mikhailov, 1939 dentro del mismo género.

Un último trabajo que es necesario destacar es el de Vachard y Tellez (1986), en el que los autores admiten 


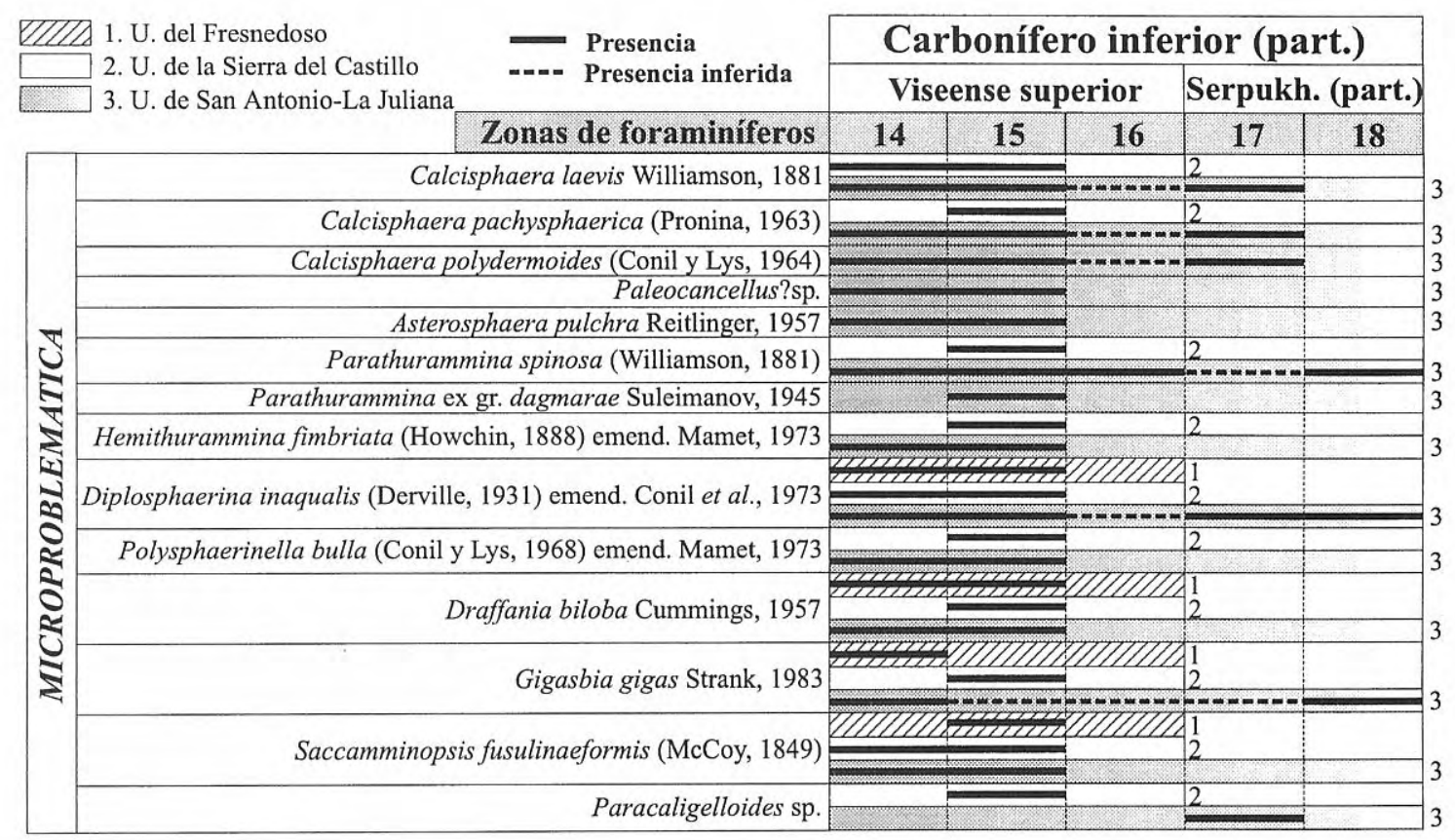

Figura 3. Distribución estratigráfica de los Microproblematica del Área del Guadiato. Stratigraphical distribution of the Microproblematica of the Guadiato Area.

que Archaesphaera no tiene poros en la pared, lo que ha provocado que haya sido clasificado en dos familias diferentes (Vachard y Tellez, 1986; Loeblich y Tappan, 1988; Vdovenko et al., 1993; etc.): Calcisphaeridae Williamson, 1881 y Tuberitinidae Miklukho-Maclay, 1958. Incluso en la clasificación que proponen Vachard y Tellez (1986) se incluye en la familia Tuberitinidae el género 2 "Diplosphaerina Derville, 1952 (= Eotuberitina Miklukho-Maclay, 1958 = Neotuberitina MiklukhoMaclay, 1958 = Quasituberitina Poyarkov, 1961)", mientras que en Calcisphaeridae incluyen el género 6 (con interrogación) "Archaesphaera Suleimanov, 1945 emend. Conil y Lys en Conil et al., 1973". Como se puede observar, ambos géneros, tal y como los definieron, representan básicamente el mismo taxón, y consideramos que debe tratarse de una errata. Nosotros interpretamos que los autores incluyen las formas sin poros en la pared y un estadio esférico (S) como Archaesphaera Suleimanov, 1945, y los ejemplares con pared porosa, en cualquier estadio (SD, D y T), en Diplosphaerina según la enmienda de Conil et al. (1973). Este último es el criterio que nosotros hemos considerado como más apropiado.

Aunque fuera del capítulo correspondiente, esta discusión de carácter taxonómico es necesaria para poder comprender las afinidades que se han supuesto para cada una de estas formas. Conil et al. (1973) y Browne y Pohl (1973) las consideraron todas como protozoos, ya que equiparan los estadios reconocidos a los existentes en muchos foraminíferos actuales durante su ciclo ontogenético. En Vachard y Tellez (1986) no queda suficientemente clara la asignación de los Tuberitinidae, ya que los autores se refieren fundamentalmente a los Calcisphaeridae, que sí son relacionados con las volvocales. Debemos interpretar que les asignan este mismo origen a los Tuberitinidae (tanto Archaesphaera como Diplosphaerina).

Polysphaerinella tiene una pared muy característica y diferenciada de la de Diplosphaerina, pero sus diferentes estadios ontogenéticos se pueden asimilar a los de Diplosphaerina, tal y como muestran Vachard y Tellez (1986) en diferentes figuras. En consecuencia, su origen debe ser el mismo que el que se interprete para Diplosphaerina.

Cummings (1957) encuentra muchas semejanzas morfológicas entre Draffania y unas estructuras de algunos equinodermos llamadas pedicellariae, aunque la pared de Draffania y la de estas estructuras es diferente. Este autor también dice que se podrían asimilar a ciertos esporangios de algunas plantas superiores, aunque descarta esta hipótesis basándose en que la calcificación de la pared es primaria en Draffania, mientras que en los esporangios de las plantas superiores es secundaria (diagenética). Otras posibles afinidades que el autor contempla son con las codiáceas, dasicladáceas y charáceas, pero las similitudes con estructuras de estas algas no son lo suficientemente consistentes.

Pocos han sido los autores que han refutado o apoyado estas hipótesis, de manera que se suelen referir a Draffania como "Problematica" (Vachard, 1977; Conil et al., 1979). Existen géneros con una pared y estructura semejantes, y que están incluidos en la familia Salebridae Bogush y Brenckle, 1982, aunque estos autores no citan ninguna afinidad posible para los géneros incluidos en la familia.

El tipo de pared laminar hace que algunos autores infieran que su posición taxonómica es cercana a los briozoos (Mamet com. per.). Este es un argumento bastante correcto desde nuestro punto de vista, ya que es muy común este tipo de microestructura en la pared de los briozoos. Otro hecho que podría corroborar este origen es 
que en las facies de montículos microbianos aparecen frecuentemente tanto Draffania como los Salebridae (Bogush y Brenckle, 1982; Gallagher, 1996); y uno de los primeros estadios de desarrollo de los "Waulsortian bank" está dominado por briozoos fenestéllidos (Lees y Miller, 1985). Sin embargo, existen otras estructuras, como son los poros y el canal central, cuya utilidad o función no se conoce en estos momentos, y que no son nada comunes en los briozoos. A pesar de lo que puedan aportar estos datos, parecen necesarios estudios más profundos sobre el género para poder conocer su posición taxonómica con una cierta verosimilitud.

Gigasbia es otro de los géneros que se caracteriza por su peculiar pared. Strank (1983) lo consideró como un Earlandidae, asignación compartida por Loeblich y Tappan (1988). Se podría considerar que Gigasbia gigas Strank, 1983 es sinónimo de Earlandia vulgaris (RauserChernoussova y Reitlinger, en Rauser-Chernoussova et al., 1936), pero la serie de planos alineados y curvados que delimitan los granos de la pared de Gigasbia no se observa en Earlandia.

Habría que considerar tres datos para inferir cual es su posición taxonómica. El primero es la morfología de la concha, idéntica a la de Earlandia, por lo que podría suponerse que Gigasbia está relacionado con éste género; aunque en este caso habría que plantearse si debe ser considerado sinónimo o si la diferenciación de pared permite considerarlo como un género independiente. En segundo lugar, su distribución paleoecológica a lo largo de la plataforma, que viene a responder al modo de vida y funcionalidad de la concha; en el Área del Guadiato se observa que los ambientes sedimentarios con una frecuencia relativa máxima de Gigasbia son idénticos a los de Earlandia, lo que apoyaría esta relación taxonómica como más probable. En tercer lugar, el tipo de pared, que no se encuentra en ningún otro tipo de foraminífero del Carbonífero. Revisando otras formas descritas en la bibliografía, Tubyphites Maslov, 1956 es el único "organismo" que se asemeja un poco, aunque se supone que la pared de éste último está originalmente constituida por células rectangulares (Mamet, 1991). Pero este "organismo" es también de origen incierto, habiéndose situado por distintos autores entre las cianobacterias (Maslov, 1956; Riding, 1991), hidrozoos (Babcock, 1977) o esponjas (Ott, en Kraus y Ott, 1968); por tanto no se llega a resolver ninguna duda.

Saccamminopsis es uno de los taxones microproblemáticos que más se ha citado en la literatura, debido a su utilidad bioestratigráfica y paleoecológica. Desde las primeras citas, se consideró como un foraminífero, debido a su similitud con Saccammina. Sin embargo, Skompski (1986) realizó una reconstrucción de Saccamminopsis como "cámaras" de un alga problemática que, debido a los agujeros de la superficie y estructura articulada, se parecería a algunas algas dasicladáceas (Mizzia). Poncet (1989) muestra secciones de "cámaras" de Saccamminopsis en las que se observa una línea negra interna con protuberancias muy bien conservadas, interpretando ésta como la calcificación de una membrana celular interna en el talo de las dasicladáceas, donde se insertan pequeñas ramas. Este autor descarta su inclusión en los protozoos ya que no existen foraminíferos actuales con membranas semejantes.

Gutteridge (1990) replica la interpretación de la línea negra hecha por Poncet (1989), considerando que puede explicarse exclusivamente mediante procesos bioestratinómicos y fosildiagenéticos, por lo que pone en duda su inclusión en las dasicladáceas o en cualquier otro grupo hasta que no sea explicado el origen de dicha línea negra.

Skompski (1993) encuentra poros "ramosos" en la pared de Saccamminopsis, aunque considera que este dato no es suficiente para asimilarlo a las algas, ya que existen foraminíferos que desarrollan este tipo de poros. Otra consideración que ha sido postulada para indicar su origen algal es la gran concentración existente en ambientes muy tranquilos (Poncet, 1989).

Pero además de resolver el problema de la línea negra, existen otros problemas que también deben ser resueltos para poder conocer cual es su posición taxonómica:

- Si fuese una dasicladácea, ¿cómo se podría explicar que en ocasiones las "cámaras" estén unidas por la abertura y la base de la siguiente, o en otros casos se unan por las aberturas, como sucede en algunos ejemplares del Guadiato?. Por tanto, parece más probable que cada una de estas "cámaras" tuviera actividad propia.

- En las cámaras que están unidas, ¿por qué la base de algunas de ellas está reabsorbida de forma que existe un "canal" central que comunica las "cámaras"? Esto sería de esperar en una dasicladácea, pero en la mayoría de las "cámaras" unidas no existe esta comunicación, insertándose la abertura de una en la pared de la base de la siguiente.

- Si el origen de las protuberancias de la línea negra es el molde de la zona donde se insertan pequeñas ramas (Poncet, 1989), ¿por qué no queda ningún "resto" o estructura identificable de estas ramas en la pared?

- ¿Cuál es la naturaleza original de la pared para que aparezca habitualmente como microesparítica?

Y todavía podrían plantearse muchas más preguntas que habría que resolver antes de proponer una posición taxonómica concreta para Saccamminopsis.

Paracaligelloides es un taxón que tradicionalmente se ha incluido en los protozoos, como un organismo con una cámara más o menos irregular, con pseudoseptos. La pared es microgranular, aunque con algunas partículas aglutinadas y donde se observan perforaciones anchas cilíndricas, que son desconocidas en los foraminíferos del Paleozoico (Armstrong y Mamet, 1977).

\section{DESCRIPCIÓN SISTEMÁTICA}

Género Calcisphaera Williamson, 1881, emend. Andrews, 1955

\section{Calcisphaera laevis Williamson, 1881} Fig. 4C

Material: 28 ejemplares. 


\section{Descripción \\ "Concha" unilocular redondeada. Diámetro de la "concha" de 70-154 $\mu \mathrm{m}$. Pared microgranular homogénea, con poros muy finos, en ocasiones apenas perceptibles (espesor de la pared 16-30 $\mu \mathrm{m}$ ).}

\section{Discusión}

Mamet (1998) figura un topotipo en el que se observan poros finos en los bordes de la pared. No se conoce con seguridad cuales son los ejemplares que utilizó Williamson (1881) para su descripción, por lo que la presencia de los poros en el material original sigue siendo una duda.

Se diferencia del resto de especies del género por su pequeño tamaño y por los poros muy finos, apenas perceptibles.

\section{Distribución}

En el Guadiato se ha encontrado en las zonas 14 y 15 en la U. Sierra del Castillo, y en las zonas 14, 15 y 17 en la U. San Antonio-La Juliana.

\section{Calcisphaera pachysphaerica (Pronina, 1963) Fig. 4D}

Material: 26 ejemplares.

\section{Descripción \\ "Concha" unilocular redondeada. Diámetro de la "concha" de 124-240 $\mu \mathrm{m}$. Pared microgranular homogénea, con poros finos (espesor de la pared de 34-60 $\mu \mathrm{m}$ ).}

\section{Discusión}

Se diferencia de $C$. laevis por tener mayor tamaño y una pared más estrecha en relación con su tamaño. Se diferencia de $C$. polydermoides (Conil y Lys, 1964) por no presentar capas microesparíticas en la pared.

\section{Distribución}

En el Guadiato se ha encontrado en la zona 15 en la U. Sierra del Castillo, y en las zonas 14,15 y 17 en la U. San Antonio-La Juliana.

\section{Calcisphaera polydermoides \\ (Conil y Lys, 1964) \\ Fig. 4E-F}

Material: 6 ejemplares.

\section{Descripción}

"Concha" unilocular redondeada. Diámetro de la "concha" de 140-202 $\mu \mathrm{m}$. Pared microesparítica con tres capas microgranulares (interna, externa y central), con un espesor de $26-40 \mu \mathrm{m}$ y atravesada por poros radiales muy marcados.

\section{Discusión}

Se diferencia del resto de especies del género por la presencia de la pared microesparítica y los poros muy marcados.

\section{Distribución}

En el Guadiato se ha encontrado en las zonas 14, 15 y 17 en la U. San Antonio-La Juliana.

\section{Género Paleocancellus Derville, 1952}

\section{Paleocancellus? sp.}

Fig. 4G

Material: 4 ejemplares.

\section{Descripción}

"Concha" unilocular redondeada. Diámetro de la "concha" de 100-160 $\mu \mathrm{m}$. Pared microesparítica con una capa interna y otra externa microgranulares, con un espesor de 38-93 $\mu \mathrm{m}$. No se han observado poros ni canales.

\section{Discusión:}

Los ejemplares se incluyen en Paleocancellus con dudas debido a que no se ha podido observar la presencia de poros o canales, ya que la capa principal de la pared es microesparítica.

\section{Distribución:}

En el Guadiato se ha encontrado en las zonas 14 y 15 en la U. San Antonio-La Juliana.

Figura 4. A. Gigasbia gigas Strank, PÑR2/1h-16, U. San Antonio-La Juliana, Zona 14 (x26). B. Parathurammina spinosa (Williamson). ADF-12-23. U. San Antonio-La Juliana, Zona 14 (x155). C. Calcisphaera laevis Williamson. BUJ14' 'bis-4, U. San Antonio-La Juliana, Zona 14 (x155). D. Calcisphaera pachysphaerica (Pronina). SSE1/1-7, U. Sierra del Castillo, Zona 15 (x155). E. Calcisphaera polydermoides (Conil y Lys). SPL7-2-1, U. San Antonio-La Juliana, Zona 17 (x155). F. Calcisphaera polydermoides (Conil y Lys). PÑR2/-13bh'-1, U. San Antonio-La Juliana, Zona 15 (x155). G. Paleocancellus? sp. PÑR5-2bis-3, U. San Antonio-La Juliana, Zona 15 (x155). H. Diplosphaerina inaequalis (Derville) emend. Browne y Pohl. CAR 1-5, U. San Antonio-La Juliana, Zona 18 (x155). I. Diplosphaerina inaequalis (Derville) emend. Browne y Pohl. ADF 2-3, U. San Antonio-La Juliana, Zona 14 (x155). J. Hemithurammina fimbriata (Howchin). BUJ 49-10, U. San Antonio-La Juliana, Zona 15 (x155). Sección oblicua. K. Hemithurammina fimbriata (Howchin). BUJ 14' '-10, U. San Antonio-La Juliana, Zona 14 (x65). L. Parathurammina ex gr. dagmarae Suleimanov. PÑR2/7-15, U. San Antonio-La Juliana, Zona 15 (x45). M. Asterosphaera pulchra Reitlinger. ADF 1-20, U. San Antonio-La Juliana, Zona 14 (x155). N. Draffania biloba Cummings. NVC/2bh-16, U. Sierra del Castillo, Zona 15 (x65). O. Polysphaerinella bulla (Conil y Lys). SPL1/4b3, U. San Antonio-La Juliana, Zona 14 (x65). P. Saccamminopsis fusulinaeformis (McCoy). SSE1/17b'-18, U. Sierra del Castillo, Zona 15 (x26). Q. Saccamminopsis fusulinaeformis (McCoy). SSE1/17b'-16, U. Sierra del Castillo, Zona 15 (x26). R. Paracaligelloides sp. VCR/0-15, U. San Antonio-La Juliana, Zona 17 (x26). 

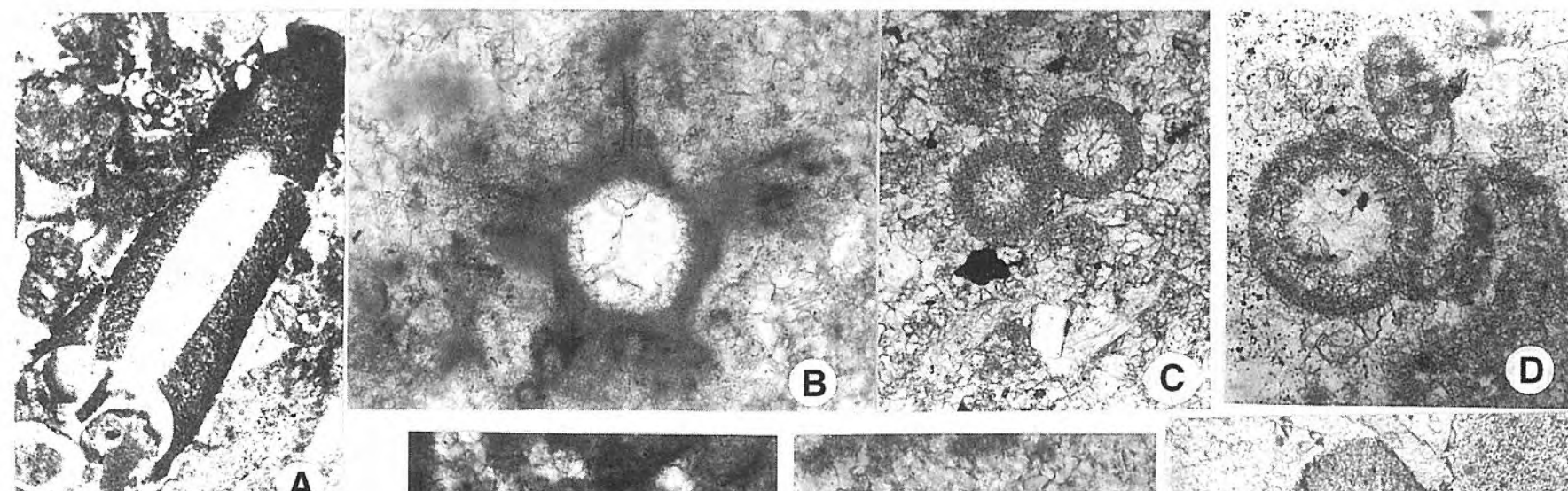

(5)

.
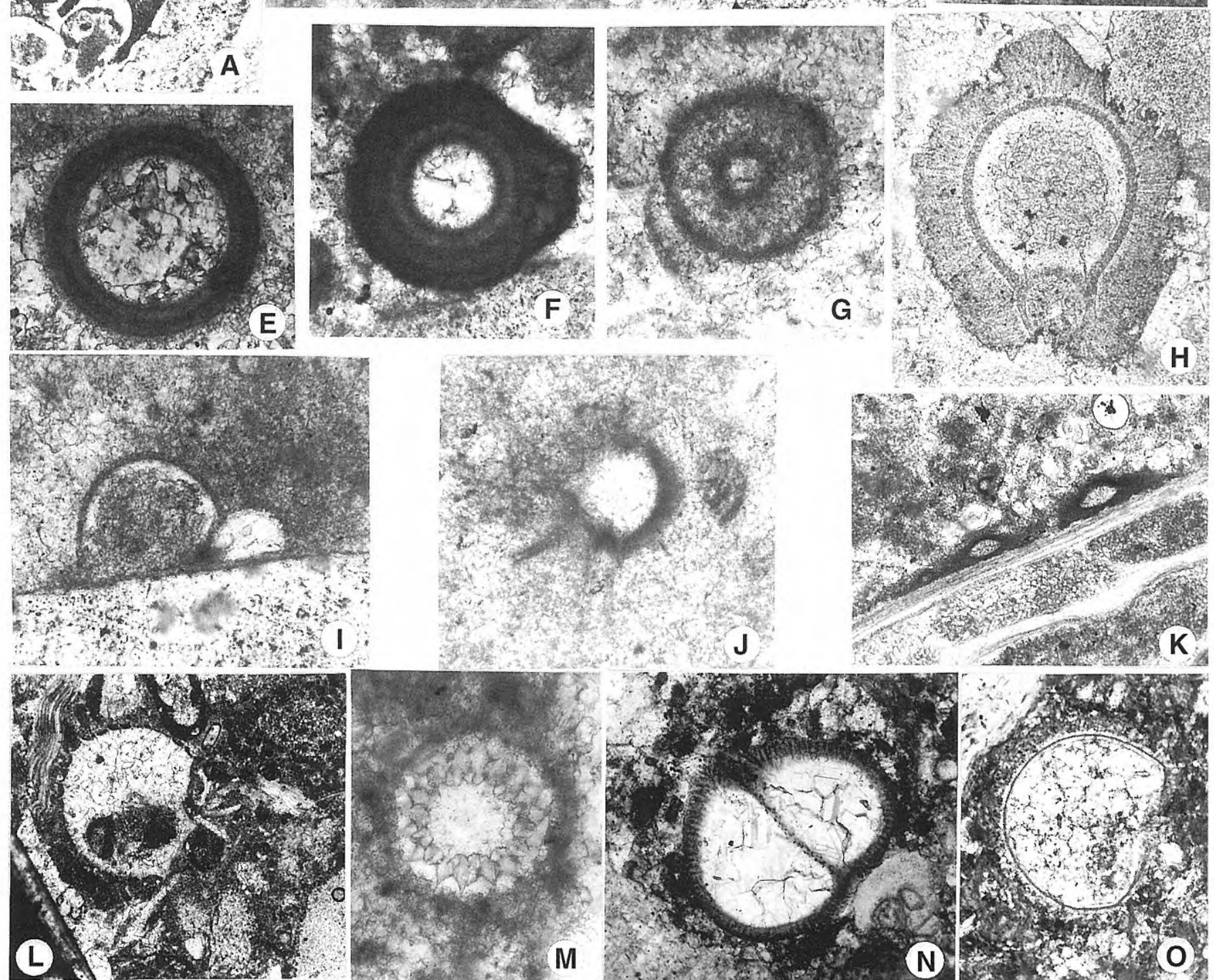

$\mathbf{J}$
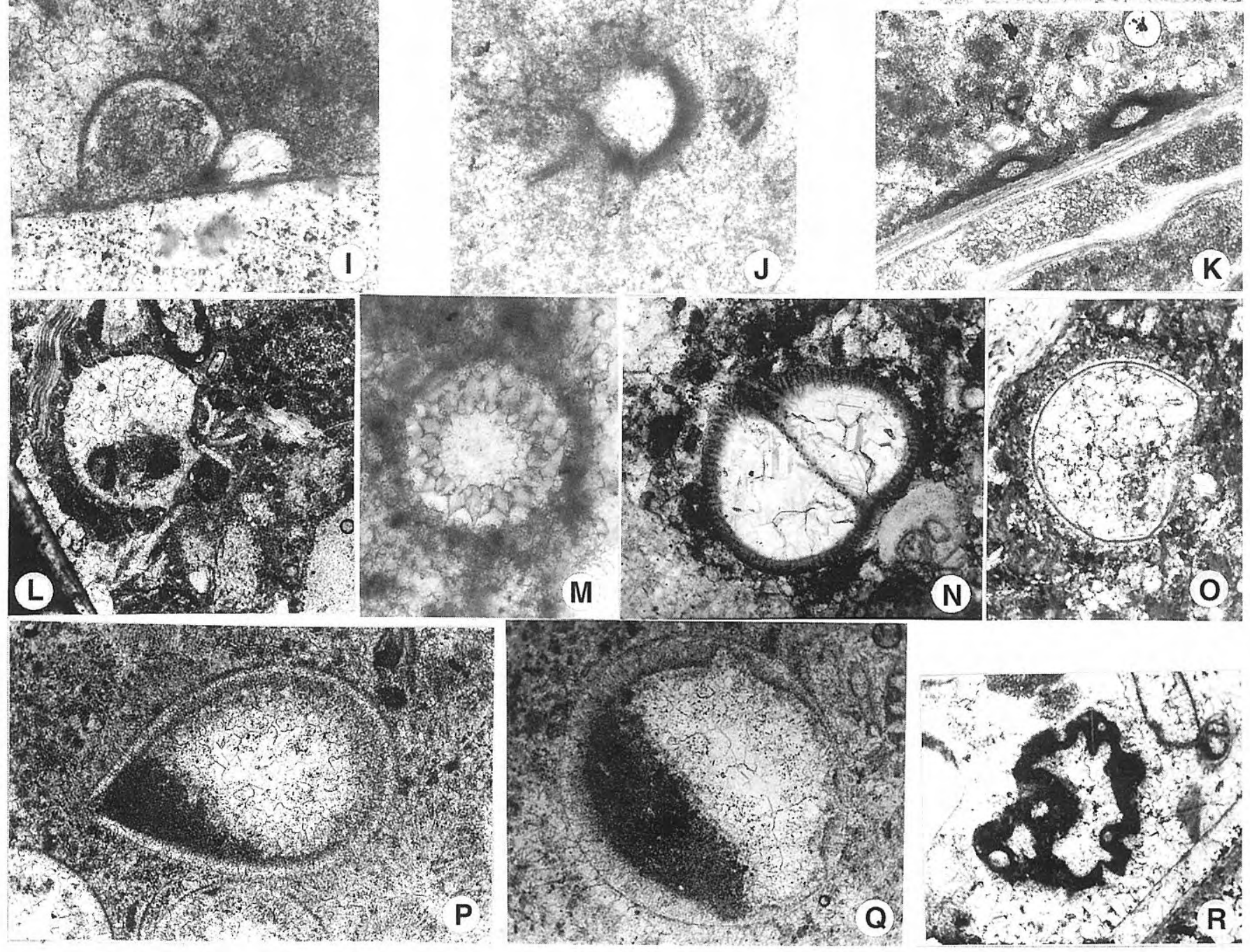
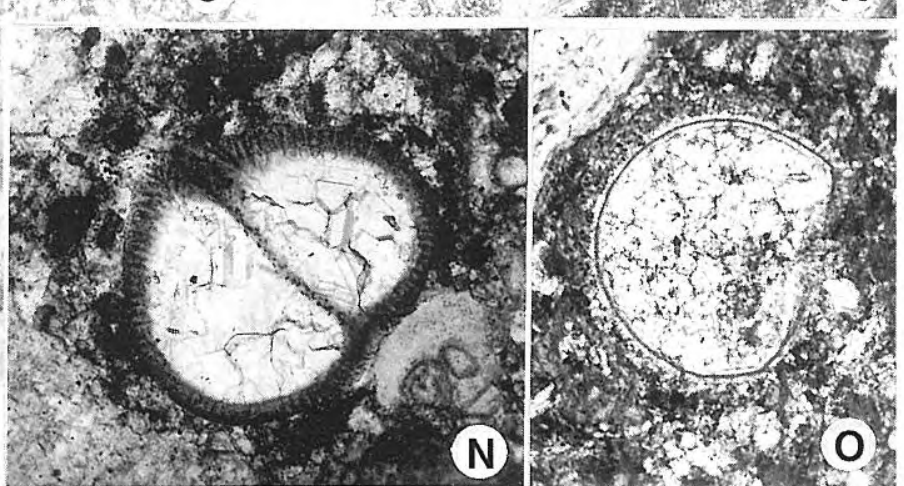

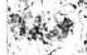

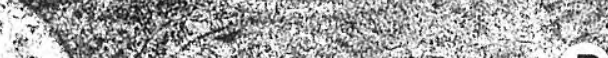
$\mathbf{P}$ 
Género Asterosphaera Reitlinger, 1957

\section{Asterosphaera pulchra Reitlinger, 1957}

Fig. 4M

Material: 22 ejemplares.

\section{Descripción:}

"Concha" redondeada con un diámetro de 134-308 $\mu \mathrm{m}$. Pared ancha (espesor de 37-77 $\mu \mathrm{m}$ ), formada por una serie de prismas de calcita anchos y dispuestos radialmente. La separación entre los prismas es mínima, pudiendo tratarse tanto de poros muy finos que comunican el agujero central con el exterior de la "concha", como deberse simplemente al relieve existente entre los prismas. Anchura de los prismas de 18-22 $\mu \mathrm{m}$. Número de prismas por sección de 20-36.

\section{Distribución}

En el Guadiato se ha encontrado en las zonas 14 y 15 en la U. San Antonio-La Juliana.

\section{Género Parathurammina Suleimanov, 1945}

\section{Parathurammina spinosa (Williamson, 1881)}

Fig. 4B

Material: 22 ejemplares.

\section{Descripción}

"Concha" poligonal con espinas con canal central. El número de espinas en sección oscila entre 4 y 9, con una longitud de 30-110 $\mu \mathrm{m}$ para un diámetro interno de la "cámara" central de 70-130 $\mu \mathrm{m}$. Pared microgranular homogénea (espesor de 5-29 $\mu \mathrm{m}$ ).

\section{Discusión}

Se diferencia del resto de especies de Parathurammina por su pequeño tamaño y bajo número de espinas por sección. Además, se diferencia de $P$. radiosphaerica Bogush y Juferev, 1962, por sus espinas en mayor número y con una longitud mucho menor.

\section{Distribución}

En el Guadiato se ha encontrado en la zona 15 en la U. Sierra del Castillo, y en las zonas $14,15,16$ y 18 en la $U$. San Antonio-La Juliana.

\section{Parathurammina ex gr. dagmarae Suleimanov, 1945}

Fig. 4L

Material: 2 ejemplares.

\section{Descripción}

"Concha" redondeada con numerosas espinas con canal central. El número de espinas en sección puede llegar a 30, con una longitud pequeña. Diámetro de la "concha" de 1100$1660 \mu \mathrm{m}$. Pared microgranular homogénea (espesor de 37-38 $\mu \mathrm{m})$.

\section{Discusión}

Aunque difiera de las especies conocidas en el grupo por un tamaño que duplica o triplica los valores de las mismas, la forma es muy similar a $P$. dagmarae, por lo que no puede situarse en otro grupo de especies.

\section{Distribución}

En el Guadiato se ha encontrado en la zona 15 en la U. San Antonio-La Juliana.

Género Hemithurammina Mamet, 1973

\section{Hemithurammina fimbriata (Howchin, 1888) emend. Mamet, 1973 \\ Fig. 4J-K}

Material: 38 ejemplares.

\section{Descripción}

"Concha" semiesférica con un disco basal saliente, del cual se proyectan espinas (con canal central). Longitud de las espinas de 30-103 $\mu \mathrm{m}$. El disco basal suele ser plano o ligeramente convexo. Diámetro máximo de la "concha" de 48-290 $\mu \mathrm{m}$, con una altura de 40-170 $\mu \mathrm{m}$. Pared microgranular homogénea, con un espesor de 4-22 $\mu \mathrm{m}$.

\section{Discusión}

Aunque en los ejemplares del Guadiato se observan algunas diferencias entre ellos (forma de la base, altura/diámetro, etc.), pensamos que representan variantes dentro de la misma especie.

\section{Distribución}

c En el Guadiato se ha encontrado en la zona 15 en la U. Sierra del Castillo, y en las zonas 14 y 15 en la U. San Antonio-La Juliana.

Género Diplosphaerina Derville, 1952 emend. Conil et al., 1973

Diplosphaerina inaequalis (Derville, 1931) emend. Conil et al., 1973

Fig. $4 \mathrm{H}-\mathrm{I}$

Material: 147 ejemplares.

\section{Descripción}

"Concha" con varios estadios ontogenéticos. En el Guadiato se han reconocido morfologías con un estadio distendido (SD), un estadio diplosférico (D) y uno tuberitínido (T). Las "conchas" en estadio distendido y diplosférico son de vida libre, y en estadio tuberitínido son incrustantes y la morfología de la base depende del bioclasto sobre el que estén fijadas. En este último estadio se encuentran en ocasiones agregados de dos y tres "individuos". El tamaño máximo de los ejemplares es de 98$375 \mu \mathrm{m}$. Pared microgranular atravesada por poros muy marcados (espesor de 4-22 $\mu \mathrm{m}$ ). 


\section{Discusión}

El reconocimiento de las formas en estado esférico (S) no es posible mediante lámina delgada; aunque suponemos que existen, no se puede llegar a precisar si son especímenes en este estadio esférico o si se trata de cortes transversales de cualquiera de los otros tres estadios.

En este trabajo consideramos que Diplosphaerina está caracterizada por tener poros en la pared, y las formas sin poros deberían ser incluidas en Archaesphaera, según fue definido por Suleimanov (1945). En consecuencia, aceptamos como válida la enmienda de Conil et al. (1973) casi en su totalidad, excluyendo de Diplosphaerina los especímenes sin poros, considerados por estos autores como los representantes más antiguos del estadio esférico (S).

\section{Distribución}

En el Guadiato se ha encontrado en las zonas 14 y 15 en la Unidad del Fresnedoso y en la U. Sierra del Castillo, y en las zonas 14, 15, 17 y 18 en la U. San Antonio-La Juliana.

\section{Género Polysphaerinella Mamet, 1973}

\section{Polysphaerinella bulla (Conil y Lys, 1968), emend. Mamet, 1973}

Fig. 40

Material: 9 ejemplares.

\section{Descripción}

Los ejemplares identificados en Guadiato presentan una "concha" con una semiesfera principal (diámetro de 280-680 $\mu \mathrm{m})$ y una serie de esférulas menores $(50-60 \mu \mathrm{m})$, dispuestas en un disco basal; por tanto presentan los estadios diplosférico(?) y tuberitínido. Pared con una delgada capa microgranular interna $(4-10 \mu \mathrm{m})$ y otra ancha y externa de composición granular-hialina (hasta $70 \mu \mathrm{m}$ ) con los bordes irregulares.

\section{Discusión}

A diferencia de Diplosphaerina, presenta en el estado diplosférico 6 a 8 esférulas o esferas polares (Mamet, 1973). No se han definido otras especies en el género.

\section{Distribución}

En el Guadiato se ha encontrado en la zona 15 en la U. Sierra del Castillo, y en las zonas 14 y 15 en la U. San Antonio-La Juliana.

\section{Género Draffania Cummings, 1957}

\section{Draffania biloba Cummings, 1957}

Fig. 4N los extremos tienen un largo cuello. El tubo central se prolonga a través del cuello. La longitud máxima de la "concha" es 384-600 $\mu \mathrm{m}$. Pared ancha (espesor de 26-76 $\mu \mathrm{m})$, con calcita laminar, y atravesada por poros.

\section{Discusión}

Los ejemplares del Guadiato tienen un tamaño menor que el habitual en $D$. biloba. Existe otra especie definida en el género, Draffania quasibiloba Fomina, 1960, con un tamaño semejante a los ejemplares del Guadiato; sin embargo, consideramos que ambas especies son sinónimas, ya que su diferenciación basada únicamente en el tamaño es insuficiente. Como ha sido demostrado en muchos foraminíferos, el tamaño final depende de la disposición de nutrientes, intensidad y duración de la iluminación (en aquellos con formas simbiontes), salinidad, tipos de mares y aportes de minerales (Murray, 1991).

\section{Distribución}

En el Guadiato se ha encontrado en las zonas 14 y 15 en la Unidad del Fresnedoso y U. San Antonio-La Juliana, y en la zona 15 en la U. Sierra del Castillo.

\section{Género Gigasbia Strank, 1983}

\section{Gigasbia gigas Strank, 1983} Fig. 4A

Material: 20 ejemplares.

\section{Descripción}

"Concha" formada por un "prolóculo" esférico saliente y una "cámara" tubular recta. Longitud máxima de la "concha" $3330 \mu \mathrm{m}$. Diámetro interno de la "cámara" tubular de 134$407 \mu \mathrm{m}$, el externo varía entre 167 y $865 \mu \mathrm{m}$. El diámetro externo del "prolóculo" varía mucho (205-851 $\mu \mathrm{m})$, proporcionalmente al tamaño de la "concha". La pared del "prolóculo" es delgada en relación con la de la "cámara" tubular, y desarrolla dos capas microgranulares muy oscuras tanto en el borde interno como en el externo. Entre estas dos capas hay otra de color grisáceo, granular; los gránulos están alineados siguiendo la curvatura de la capa microgranular interna. En la "cámara" tubular, la capa microgranular interna no está muy desarrollada, y los gránulos definen planos convexos inclinados hacia la parte externa de la "concha". El espesor de la pared oscila entre 48 y $240 \mu \mathrm{m}$.

\section{Distribución}

En el Guadiato se ha encontrado en la zona 14 en la Unidad del Fresnedoso, en la zona 15 en la U. Sierra del Castillo, y en las zonas 14 y 18 en la U. San Antonio-La Juliana.

\section{Descripción \\ "Concha" en forma de saco con dos "cámaras", separadas por un tabique con un tubo central. Las "cámaras" en uno de}

Género Saccamminopsis Sollas, 1921

Saccamminopsis fusulinaeformis (McCoy,
1849 )
Fig. $4 \mathrm{P}-\mathrm{Q}$


Material: 300 ejemplares.

\section{Descripción}

"Concha" en forma de saco, con un cuello cuya longitud oscila entre 26 y $484 \mu \mathrm{m}$. Longitud de la "cámara" de 250$2590 \mu \mathrm{m}$. Las "cámaras" pueden aparecer sueltas o unidas en series de 2 a 4 . Generalmente, la inserción se realiza entre el cuello de una y la base de la siguiente. Pared microgranular o granular con un espesor de 7-40 $\mu \mathrm{m}$. En el interior aparece una línea negra, generalmente paralela a la pared, pero en ocasiones con salientes y entrantes.

\section{Discusión}

En trabajos anteriores, algunos ejemplares de Saccamminopsis no son identificados como $S$. fusulinaeformis debido a su pequeño tamaño (i.e. Mamet, 1974). La variabilidad de tamaño encontrada en el Guadiato muestra que estos especímenes pequeños también pertenecen a $S$. fusulinaeformis.

Existe otro género que agrupa formas semejantes, Dreesenulella Vachard, 1991 (pro Baculella Conil y Dressen, en Dressen et al., 1985, ocupado), que para algunos autores es sinónimo de Saccamminopsis (Loeblich y Tappan, 1988; Skompski, 1996). Aunque estas formas son parecidas, el lapso de tiempo existente entre Saccamminopsis (Viseense superior) y Dreesenulella (Fammeniense superior) hace que Vachard (1991) los considere como géneros diferentes.

\section{Distribución}

En el Guadiato se ha encontrado en la zona 15 en la Unidad del Fresnedoso, en las zonas 14 y 15 en la U. Sierra del Castillo y U. San Antonio-La Juliana.

Género Paracaligelloides Reitlinger in Chuvashov, 1965

\section{Paracaligelloides sp.}

Fig. 4R

Material: 5 ejemplares.

\section{Descripción}

"Concha" de tamaño medio (alrededor de las $500 \mu \mathrm{m}$ ), groseramente tubular, con sus bordes, tanto interno como externo, irregulares, en los que se desarrollan proyecciones y pseudoseptos (?), marcados como constricciones. Aberturas en el final de las proyecciones. Sin "prolóculo". Pared microgranular con algún elemento aglutinado y agujeros redondeados de gran tamaño. Pared de los pseudoseptos con elementos aglutinados.

\section{Discusión}

- Los ejemplares encontrados en el Guadiato son escasos y con orientaciones muy diversas que impiden su determinación a nivel específico. Se incluyen dentro de los microproblemática debido a las perforaciones anchas y cilíndricas de la pared.

\section{Distribución}

En el Guadiato se ha encontrado en la zona 15 de la
Unidad Sierra del Castillo y en la zona 17 de la U. San Antonio-La Juliana.

\section{CONCLUSIONES}

Se ha descrito y discutido la afinidad taxonómica de 14 taxones que han sido obtenidos de los niveles carbonatados del Área del Guadiato (Figs. 1 y 2). La edad de estos afloramientos fue establecida mediante las asociaciones de foraminíferos como Viseense superior y Serpukhoviense inferior, lo que ha permitido situar las especies descritas en su contexto estratigráfico (Fig. 3).

Algunos géneros descritos pueden ser englobados en el término calcisferas (Calcisphaera, Paleocancellus y Asterosphaera); están constituidos por esferas simples, diferenciándose entre sí por la naturaleza de la pared y presencia de poros o canales. Las interpretaciones más probables de estas formas suponen que se trata de partes de dasicladáceas (Mamet, 1998) o volvocales (Kazmierczack, 1976).

Otro grupo estaría constituido por las conchas simples con espinas o parathurammínidos (Parathurammina y Hemithurammina), que se relacionan con los protozoos o con quistes calcificados de algas (Armstrong y Mamet, 1977).

Diplosphaerina y Polysphaerinella tienen una serie de estadios de crecimiento, por lo que probablemente están relacionados con los protozoos.

Gigasbia y Paracaligelloides se han considerado como protozoos (familias Earlandiidae y Caligellidae, respectivamente), pero ambos tienen una pared con ciertas peculiaridades que no se repiten en otros foraminíferos paleozoicos; habría que interpretar la naturaleza de esta pared para poder asignarles una posición taxonómica concreta.

Las estructuras de Draffania y Saccamminopsis presentan demasiados interrogantes como para precisar actualmente su posición taxonómica.

\section{AGRADECIMIENTOS}

La investigación se llevó a cabo gracias a las subvenciones de los proyectos de la DGYCIT PB91-0083 y PB96-0842. La revisión del manuscrito por la editora de esta revista y por los Dres. Bernard Mamet y Daniel Vachard ha mejorado notablemente la versión definitiva del mismo.

\section{BIBLIOGRAFÍA}

Andrews, N. R. Jr. 1955. Index of generic names of fossil plants, 1820-1950. U.S. Geological Survey Bulletin $1013,1-262$.

Armstrong, A. K. and Mamet, B. L. 1977. Carboniferous microfacies, microfossils, and corals, Lisburne Group, Arctic Alaska. U.S. Geological Survey Professional Paper, 849, 1-144.

Babcock, J. A. 1977. Calcareous algae, organic bounstones, and the genesis of the upper Capitan Limestone (Permian, Guadalupian), Guadalupe Mountains, West 
Texas and New Mexico. In: Upper Guadalupian facies Permian Reef Complex, Guadalupe Mountains, New Mexicos and West Texas (Eds. M. E. Hileman and S. J. Mazzullo). Society of Economic Paleontologists and Mineralogists, Special publication 77, 3-44.

Bogush O. I. \& Brenckle, P. L. 1982. Salebridae - una nueva familia de afinidad incierta del Carbonífero Inferior de la URSS y los Estados Unidos [en ruso]. In: Estratigrafía y paleontología del Devónico y Carbonífero (Ed. O. V. Juferev). Akademiya Nauk SSSR, Sibirsks Otdelenie, Instituta Geologii i Geofiziki, Trudy, 483, 103-118.

Bogush, O. I. \& Juferev, O. V. 1962. Estratigrafía y foraminíferos de los depósitos Carboníferos en Daraton y Talasskiy Ala-Tau [en ruso]. Akademiya Nauk SSSR, Sibirsks Otdelenie, Instituta Geologii i Geofiziki, 1-234.

Browne, R. G. and Pohl, E. R. 1973. Stratigraphy and genera of calcareous foraminifera of the Fraileys Facies (Mississippian) of Central Kentucky. Bulletin of American Paleontology, 64, 172-243.

Cayeux, L. 1929. Les calcisphères sont des algues siphonées. Comptes Rendus Académie des Sciences, 188, 594-597.

Chuvashov, B. I. 1965. Foraminíferos y algas de los sedimentos del Devónico Superior en la vertiente oeste de los Urales centrales y del sur [en ruso]. Trudy Instituta Geologicheskikh Nauk, Akademiya Nauk SSSR, Ural'skiy Filiata, 74, 3-153.

Conil, R. et Lys, M. 1964. Matériaux pour l'étude micropaléontologique du Dinantien de la Belgique et de la France (Avesnois). Partie 2, foraminifères. Mémoires du Institut de Géologie de l'Université de Louvain, 23, 1296.

Conil, R. et Lys, M. 1968. Utilisation stratigraphique des foraminifères du Dinantien. Annales de la Société Géologique de Belgique, 91, 491-558.

Conil, R., Groessens, E. et Lys, M. 1973. Étude micropaléontologique de la tranchee D'Ives-Gomezee (Tn3c-V1-V2). Bulletin de la Société belge de Géologie, 82, 201-239.

Conil, R., Longerstaey, P. J. et Ramsbottom, W. H. C. 1979. Matériaux pour l'étude micropaléontologique du Dinantien de Grande-Bretagne. Mémoires du Institut de Géologie de l'Université de Louvain, 30, 1-187.

Cózar, P. y Rodríguez, S. 1999. Propuesta de nueva nomenclatura para las unidades del Carbonífero Inferior del Sector Norte del Área del Guadiato (Córdoba). Boletín Geológico y Minero, 110, 237-254.

Cózar Maldonado, P. 1998. Bioestratigrafía con foraminíferos del Carbonífero Inferior del sector Norte del Área del Guadiato (Córdoba). Tesis doctoral de la Facultad de Ciencias Geológicas, Universidad Complutense de Madrid, 1-590 (inédita).

Cummings, R. H. 1957. A problematic new microfossil from the Scottish Lower Carboniferous. Micropaleontology, 3, 407-409.

Derville, H. 1931. Les Marbres du Calcaire Carbonifère en Bas-Boulonnais. Strasbourg, Boehm editeur, 1-322.

Derville, H. 1952. A propos de Calcisphères (Rectificatión). Comptes Rendus Somaire de la Société Géologique de
France, 6 ${ }^{\mathrm{a}}$ série, 2, 236-237.

Dreesen, R., Bless, M. J. M., Conil, R., Flajs, G. and Laschet, C. 1985. Depositional environment, paleoecology and diagenetic history of the "Marbre Rouge a crinoides de Baelen" (Late upper Devonian, Verviers Synclinorium, Eastern Belgium). Annales de la Société Géologique de Belgique, 108, 311-359.

Fomina, E. V. 1960. Sobre algunos foraminíferos de la Cuenca de Moscú [en ruso]. In: Materialii Geologicheskikh Polez Iskopanii Central Rayon Europ Chasti SSSR, 114-119.

Gallagher, S. J. 1996. The stratigraphy and cyclicity of the late Dinantian platform carbonates in parts of southern and western Ireland. In: Recent advances in Lower Carboniferous Geology (Eds. P. Strogen, I. D. Somerville and G. Ll. Jones). Geological Society Special Publication 107, 239-251.

Groves, J. R. 1987. Evidence for syzygy in Late Paleozoic calcareous foraminifers: early origin for an "advanced" reproductive strategy. Courier Forschungs-Institut Senckenberg, 98, 5-10.

Groves, J. R. 1988. Insolentitheca and Insolentithecinae, inappropiate names for Paleozoic syzygial cysts. Journal of Foraminiferal Research, 18, 302-303.

Gutteridge, P. 1990. Comment on Saccamminopsis affinities. Lethaia, 23, 222.

Howchin, W. 1888. Aditions to the knowledge of the Carboniferous foraminifera. Royal Microscopical Society Journal, 2, 533-545.

Judd, T. 1877. Reference to the nature of calcispheres. In: On the structure and affinities of the genus Siphonia (Sollas, W. J.). Geological Society of London Quarterly Transsactions, 33, 790-835.

Kazmierczack, J. 1975. Colonial Volvocales (Chlorophyta) from the Upper Devonian of Poland and their paleoenvironmental significance. Acta Paleontologica Polonica, 20, 73-85.

Kazmierczack, J. 1976. Volvocacean nature of some Paleozoic nonradiosphaerid calcispheres and parathuramminid "Foraminifera". Acta Paleontologica Polonica, 21, 245-258.

Kazmierczack, J. 1981. The biology and evolutionary significance of Devonian Volvocaceans and their Precambrian relatives. Acta Paleontologica Polonica, 26, 299-337.

Kraus, O. und Ott, E. 1968. Eine ladinische Riff-fauna in Dobratsch-Gipfelkalk (Kärnten, Österreich) und Bemerkungen zum Faciesvergleich von Nordalpen and Druzug. Mitt Bayer Staatsamml Paläontologie Historiche Geologie, 8, 263-298.

Lees, A. and Miller, J. 1985. Facies variation in Waulsortian buildups. Part 2. Mid-Dinantian buildups from Europe and North America. Geological Journal, 20, 159-180.

Loeblich, A. R. and Tappan, H. 1964. Part C. Protista 2: Sarcodina chiefly "Thecamoebians" and Foraminiferida. In: Treatise on invertebrate Paleontology (Ed. R.C. Moore). The Geological Society of America. Vol. 1. 1-900.

Loeblich, A. R. and Tappan, H. 1988. Foraminiferal genera and their classification. Van Nostrand Reinhold Company, New York, 1-970. 
Mamet, B. L. 1973. Microfaciès Viséens du Boulonnais (Nord, France). Revue de Micropaléontologie, 16, 101124.

Mamet, B. L. 1974. Une zonation par foraminifères du Carbonifère inférieur de la Téthys occidentale. Comptes Rendus 7 Congrès International de Stratigraphie et Géologie du Carbonifère, 3, 391-408.

Mamet, B. L. 1991. Carboniferous calcareous algae. In: Calcareous algae and stromatolites (Ed. R. Riding). Springer-Verlag Berling-Heidelber, 370-451.

Mamet, B. L. 1998. A late Devonian microfossil with dasyclad algae affinities form northwestern Australia. Alcheringa, 22, 21-28.

Mamet, B. L. et Boulvain, F. 1992. Microflore des montícules micritiques frasniens F2j de Belgique. Revue de Micropaléontologie, 35, 283-302.

Maslov, V. P. 1956. Algas calcáreas fósiles de la URSS [en ruso]. Akademiya Nauk SSSR, Instituta Geologicheskikh Nauk, 160, 1-322.

McCoy, F. 1849. On some new genera and species of Paleozoic corals and foraminifera. Annals and Magazine of Natural History, ser. 2, 3, 134.

Mikhailov, A. V. 1939. Sobre las características de los géneros de foraminíferos del Carbonífero Inferior. In: Los depósitos del Carbonífero inferior de la zona noroeste de la cuenca de Moscú (Ed. S. F. Maljavskin) [en ruso]. Sbornik Leningradskogo Geologischeskogo Upravleniya, Glavnoe Geologicheskoe Upravlenie, 3, 47-62.

Miklukho-Maclay, A. D. 1958. Una nueva familia de foraminíferos - Tuberitinidae Miklukho-Maclay, Fam. nov. [en ruso]. Akademiya Nauk SSSR Voprosy Mikropaleontologii, 2, 130-135.

Murray, J. W. 1991. Ecology and Palaeoecology of benthic foraminifera. Longman, Harlow, 1-397.

Poncet, J. 1989. New data about the taxonomic position of Saccaminopsis. Lethaia, 22, 425-429.

Poyarkov, B. V. 1961. Foraminíferos. In: Estratigrafía y nuevas especies de foraminíferos del Tournaisiense de los Montes de Borkoldoi (Tian Chan) (Eds. M.M. Purkin, B.V. Poyarkov y V.M. Rozanc) [en ruso]. Akademiya Nauk Kirgizskoy SSR, Izvestiya Seriya Nauk Natsional'nyi, 3, 15-36.

Pronina, T. V. 1963. Foraminíferos del Carbonífero de la serie de Berezovo en la vertiente este de los Urales del sur. In: Documentos sobre problemas en estratigrafía 7 , Estratigrafía y fauna del Paleozoico de los Urales [en ruso]. Trudy Instituta Geologicheskikh Nauk, Akademiya Nauk SSR, Ural'skiy Filial, 65, 119-176.

Rauser-Chernoussova, D. M., Beljaev, G. M. \& Reitlinger, E. A. 1936. Foraminíferos del Paleozoico Superior de la región de la Pechora (NO de los Urales) [en ruso]. Trudy Polyarnoy Komisii Akademiya Nauk SSSR, 28, 159-232.

Reitlinger, E. A. 1957. Esferas del Devónico de la Plataforma Rusa [en ruso]. Doklady Akademiya Nauk SSSR, 115, 774-776.

Riding, R. 1991. Calcified Cyanobacteria. In: Calcareous algae and stromatolites (Ed. R. Riding), Springer-Verlag Berling-Heidelber, 55-87.

Rupp, A. W. 1966. Origin, structure and environmental significance of recent and fossil Calcispheres. Geological
Society America program 1966, Annals Meetings, San Francisco, 186.

Skompski, S. 1986. Upper Viséan calcareous algae from the Lublin Coal Basin. Acta Geologica Polonica, 36, 251280.

Skompski, S. 1993. Shell structure and affinity of the Carboniferous microproblematicum Saccamminopsis. Lethaia, 26,147-152.

Skompski, S. 1996. Stratigraphic position and facies significance of the limestone bands in the subsurface Carboniferous succession of the Lublin Upland. Acta Geologica Polonica, 46, 171-268.

Sollas, W. J. 1921. On Saccammina carteri Brady, and the minute structure of the foraminiferal shell. Quarterly Journal of the Geological Society of London, 77, 193212.

Strank, A. R. E. 1983. New stratigraphically significant foraminifera from the Dinantian of Great Britain. Palaeontology, 26, 435-442.

Suleimanov, I. S. 1945. Algunas especies de pequeños foraminíferos del Tournaisiense de Ishimbayevo de las regiones petrolíferas [en ruso]. Doklady Akademiya Nauk SSSR, 48, 124-127.

Vachard, D. 1977. Étude stratigraphique et micropaléontologique (algues et foraminifères) du Viséen de la Montagne Noire (Hérault, France). Mémoires du Institut de Géologie de l'Université de Louvain, 29, 111-195.

Vachard, D. 1991. Parathuramminides et moravamminides (Microproblematica) de l'Emsien supérior de la Formation Moniello (Cordilleres Cantabriques, Espagne). Revue de Paléobiologie, 10, 255-299.

Vachard, D. 1994. Foraminifères et moravamminides du Givetien et du Frasnien du Domaine ligerien (Massif Armoricain, France). Paleontographica Abteilungen, 231, 1-92.

Vachard, D. y Téllez, C. 1986. El género Polyderma y nuevas soluciones a la sistemática de las calcisferas, microfósiles problemáticos del Paleozoico. Revista del Instituto Mexicano del Petróleo, 18, 6-44.

Vdovenko, M. V., Rauser-Chernoussova, D. M., Reitlinger, E. A. \& Sabirov, V. A. 1993. Reference on systematic of small Paleozoic foraminifera (with the exception of the endothyroids and Permian multichambered lagenoids) [en ruso]. Trudy, Geological Institut, Russian Academy of Sciences, Commission of Micropaleontology, "Nauka", Moscow, 1-128.

Villa, E. y Sánchez de Posada, L. C. 1991. Nota sobre la posible existencia de quistes sicigiales en foraminíferos del Carbonífero Cantábrico. Revista Española de Paleontología, Número extraordinario, 185-195.

Williamson, W. C. 1881. On the organization of the fossil plants of the Coal Measure (Part X). Including an examination of the supposed Radiolarians of the Carboniferous rocks. Royal Society London Philosophical Transaction, 171, 493-539. 